\title{
Aplicación del modelo de regresión múltiple para la interpolación de las temperaturas y precipitaciones de la península ibérica y las Islas Baleares
}

\section{Application of the multiple regression model for the interpolation of temperatures and rainfall at the Iberian Peninsula and the Balearic Islands}

\author{
Fernando Pardo Navarro ${ }^{1}$
}

\section{RESUMEN}

Se han elaborado mapas de precipitación mensual y temperatura media mensual para la península ibérica y Baleares mediante la interpolación de los valores de 3736 estaciones pluviométricas y 1626 estaciones termométricas, con software estadístico y de sistemas de información geográfica libres. El método utilizado fue la regresión múltiple con interpolación de residuos. Distintas variables no utilizadas en modelos anteriores mejoraron el ajuste de las regresiones. Entre ellas la altitud y pendiente medias en el caso de la precipitación y la radiación real en la temperatura. La validación de los modelos con el $30 \%$ de los datos presenta errores similares a los obtenidos en trabajos previos. La comparación de los datos del modelo climático con los de los 60 Observatorios Meteorológicos Principales españoles para los tres últimos periodos climáticos referenciales (1961-1990, 1971-2000 y 1981-2010), muestra que el modelo, en líneas generales, sobrestima las precipitaciones y subestima las temperaturas actuales debido a los cambios climáticos recientes.

Palabras Clave: Baleares; interpolación; modelo de regresión múltiple; península ibérica; precipitación; temperatura.

1 IES Enrique Tierno Galván, Calle Agustinos, s/n, 28914, Leganés, Madrid. E-mail: fernando.pardonavarro@educa.madrid.org ORCID iD: http://orcid.org/ 0000-0002-4308-617X 


\section{Abstract}

Monthly precipitation and temperature maps for the Iberian Peninsula and Balearic Islands were calculated using different free statistical and GIS software and the data from 3736 rainfall and 1626 temperature meteorological stations. The method used was multivariate regression with residuals interpolation. Different variables not used in earlier studies improve the regression models: mean altitude and slope for precipitation models and real radiation for temperature models. The validation of models with 30 percent of the data showed errors similar to previous works. The climatic model data were compared with the data from the 60 Meteorological Main Observatories from Spain for the three last Standard Reference Periods (1961-1990, 1971-2000 and 1981-2010). The model overestimate current precipitation and underestimate current temperatures due to to recent climate changes..

KEY words: Balearic; interpolation; Iberian Peninsula; multiple regression model; precipitation; temperature.

Cómo citar este artículo/Citation: Pardo Navarro, Fernando (2017): “Aplicación del modelo de regresión múltiple para la interpolación de las temperaturas y precipitaciones de la península ibérica y las Islas Baleares", Estudios Geográficos, LXXVIII/283, pp. 493-522

\section{INTRODUCCIÓN}

Muchos procesos biológicos y físicos están influidos por el clima, por lo que distintas ciencias utilizan datos climáticos en sus investigaciones. Como la resolución de los datos suele ser baja, una importante tarea de la climatología ha sido suministrar información sobre el clima donde no existen registros meteorológicos, siendo necesario para ello usar algún método de interpolación (Tveito, 2008). Estos presentan diferencias en sus bases conceptuales y formulación matemática (Daly, 2006; Hengl, 2009). Algunos métodos utilizan datos externos (geográficos y topográficos), habiendo sido de gran utilidad los sistemas de información geográfica (SIG) para combinar diferentes parámetros y producir estimaciones de variables climáticas con alta resolución espacial (Chapman y Thornes, 2003).

La precisión de los diferentes métodos de interpolación depende de las características del área de estudio, la escala espacial a la que se construyen los mapas y las variables climáticas calculadas, entre otros factores (Daly, 2006). No obstante, en áreas de topografía heterogénea, los métodos que usan variables externas recogen mejor la riqueza espacial del clima y tienen errores de predicción menores (Hevesi et al., 1992; Ishida y Kawashima, 1993; MartínezCob, 1996; Goodale et al., 1998). En este sentido, un método comúnmente 
utilizado en áreas irregulares es la regresión múltiple con interpolación de residuos (Agnew y Palutikof, 2000; Kyriakidis et al., 2001; Brown y Comrie, 2002; Vicente-Serrano et al., 2003; Guan et al., 2005; Perry y Hollis, 2005; Ninyerola et al., 2007a; Ninyerola et al., 2007b). La regresión recoge la relación entre las variables calculadas y distintas variables independientes. Los residuos representan la variabilidad que no queda recogida por la relación determinista representada por las funciones de regresión (Hengl et al., 2007; Hengl, 2009). La inclusión de los residuos en los modelos climáticos normalmente mejora las estimaciones (Agnew y Palutikof, 2000; Brown y Comrie, 2002; VicenteSerrano et al., 2003; Perry y Hollis, 2005; Ninyerola et al., 2007a; Ninyerola et al., 2007b). La selección de las variables ambientales más adecuadas para la predicción de las variables climáticas es clave en este método de interpolación (Agnew y Palutikof, 2000).

El objetivo de este trabajo es obtener mapas mensuales de precipitación y temperaturas medias de la península ibérica e islas Baleares e investigar qué factores geográficos y topográficos juegan un papel importante en la elaboración del modelo climático. Para ello se utilizarán software estadístico y sistemas de información geográfica de uso libre. Se usará una regresión lineal multivariante seguida de la interpolación de residuos mediante métodos geoestadísticos. Los mapas finales se construirán sumando la superficie obtenida con la regresión multivariante y los mapas de los residuos interpolados (figura 1).

FIGURA 1

RESUMEN DE LA METODOLOGÍA

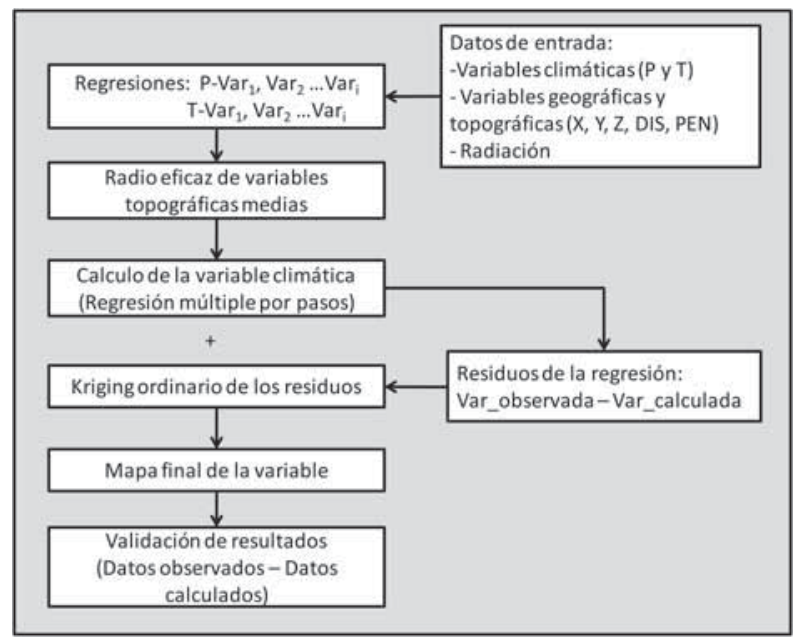

Estudios Geográficos, Vol. LXXVIII, 283, pp. 493-522, julio-diciembre 2017 ISSN: 0014-1496, eISSN: 1988-8546, doi: 10.3989/estgeogr.201717 
Metodología

El área de estudio abarcó la península ibérica, que ocupa un total de 5581471 $\mathrm{km}^{2}$, y las Islas Baleares, que cubren $4992 \mathrm{~km}^{2}$. El territorio, situado en la transición de la zona de influencia de las altas presiones subtropicales y los vientos del oeste de la zona templada, presenta unas características climáticas extensamente estudiadas por distintos autores (Capel, 2000; Font Tullot, 2000; Martín Vide y Olcina, 2001).

Las regresiones múltiples de las variables climáticas frente a distintos factores se calcularon con el software OpenStat (Miller, 2013). Los mapas de las variables obtenidas por regresión múltiple se crearon con el SIG de uso libre GRASS (acrónimo en inglés de «Geographic Resources Analysis Support System») (Neteler y Mitasova, 2004) y los residuos interpolados de cada variable con el SIG ILWIS (acrónimo de «Integrated Land and Water Information System») (ITC, 2001).

Una muestra control aleatoria del $30 \%$ de los datos fue separada para comprobar la validez del modelo con datos independientes a lo largo de toda la zona de estudio (figura 2) (Tveito, 2008). Para la construcción de los mapas finales se utilizaron todas las estaciones meteorológicas. Los mapas resultantes tendrán un nivel de error menor o, a lo sumo, igual que los mapas obtenidos utilizando solamente el $70 \%$ de las estaciones meteorológicas (Ninyerola et al., 2000b; Ninyerola et al., 2007a), al incluir un mayor número de estaciones que cubrirán mucho mejor toda la zona estudiada.

FIGURA 2

MAPAS DE LAS ESTACIONES PLUVIOMÉTRICAS Y TERMOMÉTRICAS
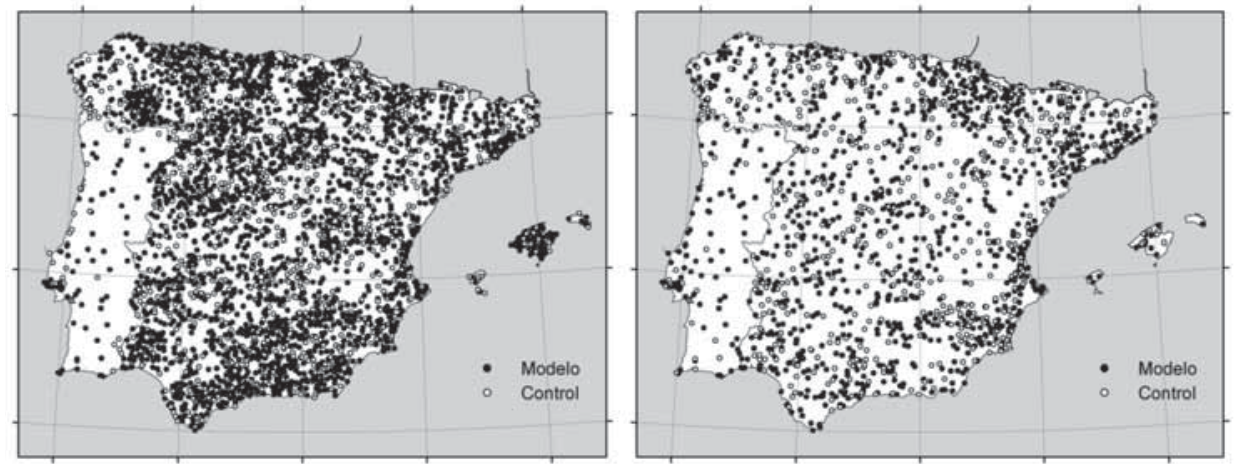

Estudios Geográficos, Vol. LXXVIII, 283, pp. 493-522, julio-diciembre 2017 ISSN: 0014-1496, eISSN: 1988-8546, doi: 10.3989/estgeogr.201717 
También se compararon los valores climáticos calculados con los de los Observatorios Principales españoles (excepto Canarias) para los tres últimos periodos climáticos referenciales: 1961-1990, 1971-2000 y 1981-2010 (INM, 2004), para analizar las diferencias del modelo con el clima más reciente. Corresponden a esta red de Observatorios 60 estaciones que coinciden en los tres periodos referenciales.

\section{Datos climáticos y topográficos}

Todas las variables utilizadas en los modelos multivariantes fueron obtenidas de bases de datos públicas. Los datos de temperaturas y precipitaciones de las provincias españolas se obtuvieron del Sistema de Información Geográfica de Datos Agrarios del Ministerio de Agricultura (http://sig.magrama.es/sigal; fecha de consulta: 9/02/2009). Los datos portugueses provienen de la base de datos AGRIBASE (http://agricultura.isa.utl.pt/agribase_temp/solos/default. asp; fecha de consulta: 10/09/2013). Las series utilizadas corresponden al periodo 1960-2003. Solo se emplearon las estaciones pluviométricas con más de 20 años de datos y las termométricas con más de 15 años, considerando que estos periodos suponen el mejor compromiso espacio-temporal. El uso de un periodo de 30 años, recomendado por la Organización Meteorológica Mundial, dejaría grandes áreas sin cubrir y la interpolación produciría unos resultados menos precisos (Willmott et al., 1996; Kurtzman y Kadmon, 1999; Ninyerola et al., 2007a; Ninyerola et al., 2007b). La base de datos final utilizada comprende 3736 estaciones pluviométricas y 1626 estaciones de temperatura que fueron geo-referenciadas en una cobertura digital con coordenadas UTM, en el uso 30N (Sistema Geodésico ETRS-89).

La representatividad de la red de estaciones, que normalmente se encuentra sesgada hacia las zonas de baja altitud y más pobladas (Tveito, 2008), fue analizada comparando la distribución de altitudes de la zona de estudio con la distribución de altitudes de las estaciones (figura 3). Se observa que el porcentaje de estaciones a mayor altitud es menor que el de territorio. La consecuencia de este sesgo es la extrapolación de las variables climáticas fuera del área válida del modelo, un problema frecuente en la interpolación de datos climáticos en áreas de montaña (Daly, 2006). 
FIGURA 3

DISTRIBUCIÓN DE LAS ALTITUDES DEL ÁREA Y DE LAS ESTACIONES

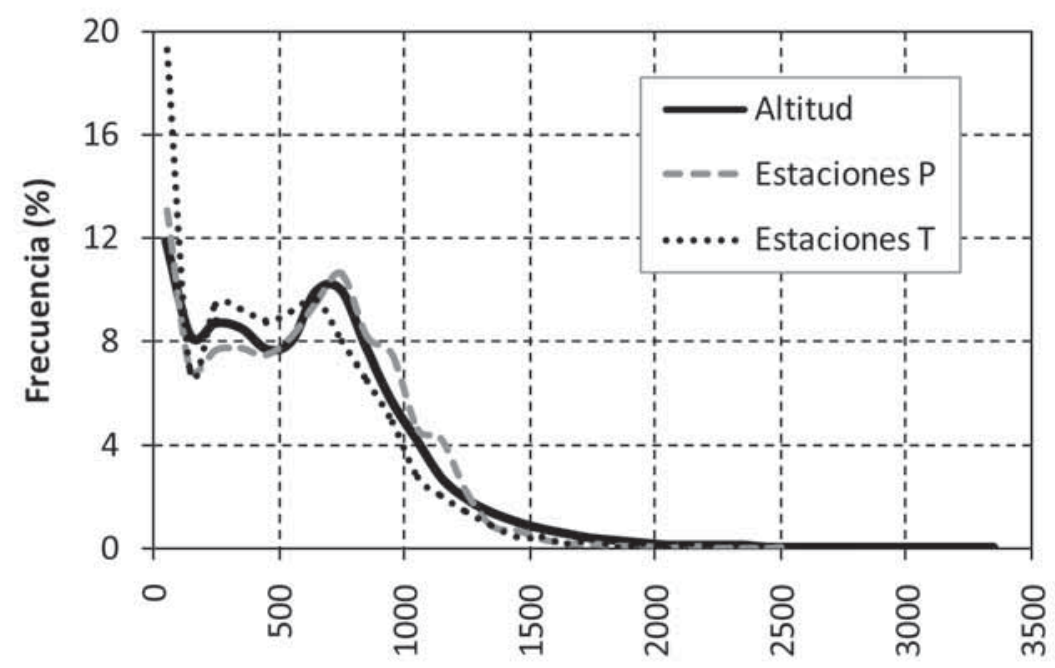

Altitud (m)

Las variables topográficas se estimaron para cada celda del mapa con una resolución de 200 × 200 m. El Modelo Digital de Elevaciones (MDE) proporcionado por el CGIAR-CSI ( «Consortium for Spatial Information» del «Consultative Group for International Agricultural Research»), disponible en http://srtm.csi.cgiar.org/ (fecha de consulta: 20/09/2013) constituyó la base de los datos espaciales de referencia.

Se utilizó un mapa vectorial de la línea de costa para diferenciar las tierras emergidas de las zonas marinas. Estas últimas se transformaron en datos tipo raster con la misma resolución que el modelo de elevaciones y un valor asignado de cero metros. El mapa de costas fue obtenido de la base de datos de alta resolución GADM, acrónimo en inglés de «Database of Global Administrative Areas», http://www.gadm.org/ (fecha de consulta: 13/10/2013). Las coordenadas geográficas, longitud (X) y latitud (Y) fueron asignadas a cada punto del mapa raster del área de estudio y utilizadas como variables independientes de las regresiones. 


\section{Radiación}

La radiación solar se incluyó en los modelos de las temperaturas al ser una variable directamente relacionada con la energía que llega a la superficie del terreno, afectando a sus características térmicas. Se utilizaron tres colecciones de datos de radiación (figura 4). La primera, la radiación potencial (Rp), fue obtenida del modelo de radiación solar del SIG GRASS, que corresponde a la radiación con cielo despejado y es dependiente exclusivamente del ángulo solar de incidencia, el relieve y la latitud.

La segunda colección de datos, radiación real $(\mathrm{Rr})$, se obtuvo de la base de datos CMSAF (The Satellite Application Facility on Climate Monitoring, http://www.cmsaf.eu; fecha de consulta: 28/07/2014). Se utilizaron los parámetros SIS (Surface Incoming Solar radiation) y SID (Surface Incoming Direct radiation), correspondientes respectivamente a la radiación solar total y a la radiación directa sobre una superficie horizontal (la diferencia entre ambas constituye la radiación difusa). Los datos, suministrados con una resolución de $3 \mathrm{~km}$, fueron reescalados a la misma resolución que el resto (200 m). La base de datos cubre un periodo de 22 años (1983-2005), derivándose del radiómetro MVIRI (Meteosat Visible Infra-Red Imager), de los satélites Meteosat de primera generación. Las medias mensuales de todo el periodo de registro fueron calculadas y utilizadas en los modelos de regresión.

La tercera colección de datos de radiación (Rc) combina las dos anteriores (figuras 4 y 5), corrigiendo la radiación real con un factor topográfico. Este factor, similar al utilizado por (Xin et al., 2007), fue igual a la relación entre la radiación potencial sobre una superficie horizontal y la radiación potencial sobre la superficie real, con una pendiente, orientación y posición topográfica dadas. Para su cálculo, primero se estimó la radiación potencial sobre una superficie plana con el SIG GRASS, utilizando como datos de entrada la pendiente y exposición de una superficie plana (ambas con valor cero), además de los datos de altitud y latitud. Después se calculó la relación entre la radiación potencial en el terreno real y en la superficie plana. Los valores obtenidos fueron multiplicados por la radiación real, correspondientes a una superficie horizontal, para corregirlos con el efecto de la topografía. El cálculo de este tercer tipo de radiación se hizo independientemente para las radiaciones directa y difusa (que después fueron sumadas), ya que la proporción relativa de ambas es muy variable a lo largo de los meses, debido principalmente a las diferencias en la nubosidad. 
FIGURA 4

MAPAS DE TRES TIPOS DE RADIACIÓN PARA EL MES DE ENERO
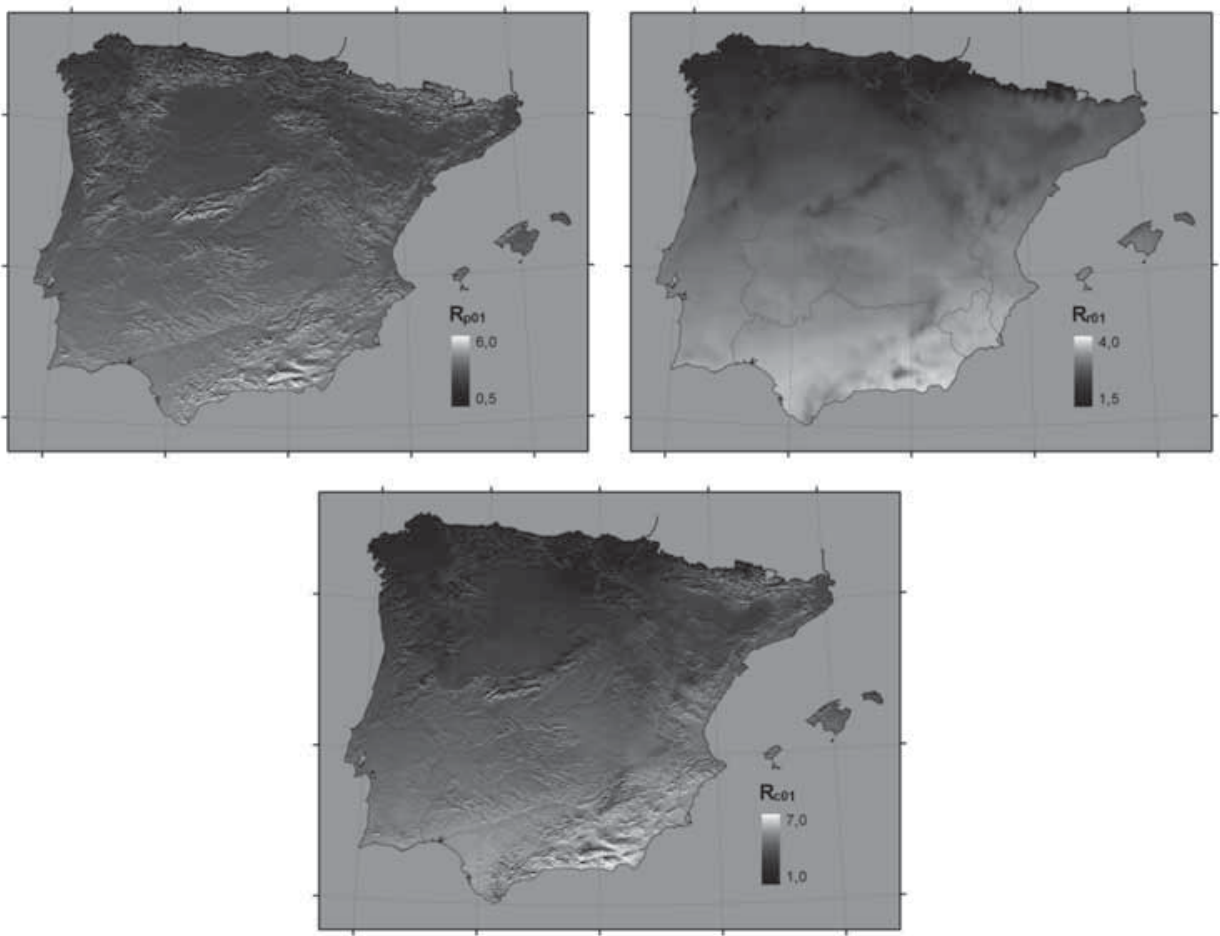

FIGURA 5

METODOLOGÍA PARA EL CÁLCULO DE LA RADIACIÓN CORREGIDA

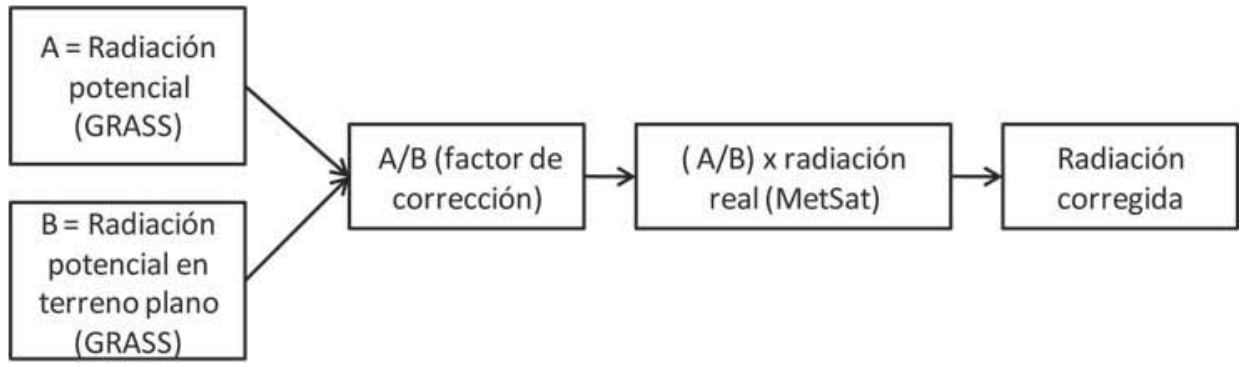

Estudios Geográficos, Vol. LXXVIII, 283, pp. 493-522, julio-diciembre 2017 ISSN: 0014-1496, eISSN: 1988-8546, doi: 10.3989/estgeogr.201717 


\section{Modelos de regresión múltiple}

Se compararon modelos de regresión de complejidad creciente para cada variable dependiente de precipitación y temperatura. La normalidad de cada variable fue analizada, aplicando alguna transformación cuando no cumplieron el test de normalidad (Miller, 2013) (tabla 1). De las variables dependientes solo la precipitación necesitó una transformación para seguir una distribución normal, como se ha observado en otros trabajos (Phillips et al., 1992; Daly et al., 1994; Nalder y Wein, 1998).

TABLA 1

VARIABLES UTILIZADAS EN LOS MODELOS DE REGRESIÓN

\begin{tabular}{l|c}
\hline Variable Independiente & Abreviatura \\
\hline Longitud UTM (m) & $\mathrm{X}^{\mathrm{a}}$ \\
\hline Latitud UTM (m) & $\mathrm{Y}^{\mathrm{a}}$ \\
\hline Altitud (m) & $\mathrm{Z}^{\mathrm{a}}$ \\
\hline Altitud media para un radio $\mathrm{x}_{\mathrm{i}}$, donde $\mathrm{x}_{\mathrm{i}}$ vale 4,8,15,30 o $60 \mathrm{~km}$ & $\mathrm{Z}_{\mathrm{x}}^{\mathrm{a}}$ \\
\hline Pendiente media $(\%)$ para un radio $\mathrm{x}_{\mathrm{i}}$ igual a $4,8,15,30$ o $60 \mathrm{~km}$ & $\mathrm{PEN}_{\mathrm{x}}^{\mathrm{b}}$ \\
\hline Distancia a la costa más próxima $(\mathrm{m})$ & $\mathrm{DIS}^{\mathrm{a}}$ \\
\hline Radiación mensual media $\left(W h . \mathrm{m}^{-2} \cdot\right.$ día $\left.^{-1}\right)$ & $\mathrm{RAD}^{\mathrm{b}}$ \\
\hline
\end{tabular}

a Variable transformada mediante raíz cuadrada

${ }^{\mathrm{b}}$ Variable transformada mediante logaritmo natural

En todos los análisis se aplicó el método de regresión lineal múltiple por pasos hacia delante, que permite la exclusión de las variables independientes estadísticamente no significativas $(\alpha>0.05)$. Finalmente se seleccionaron las regresiones con el menor error medio absoluto (siglas MAE, del inglés «mean absolute error») y el máximo coeficiente de determinación $\left(R^{2}\right)$.

En los modelos multivariantes de la precipitación se utilizaron como variables independientes la altitud, que muestra normalmente una correlación positiva con la precipitación (Smith, 1979; Daly et al., 1994), y la pendiente, 
que tiene un efecto positivo debido a que el enfriamiento adiabático de las masas de aire que cruzan una elevación del terreno es más rápido a mayor pendiente (Barry, 1992).

También se utilizaron los valores medios para diferentes radios $(2,4,8,15$, 30 y $60 \mathrm{~km}$ ) de estas dos variables topográficas. Esta elección se hizo teniendo en cuenta que los efectos de la topografía en la precipitación a veces se manifiestan a escalas de varias decenas de kilómetros (Daly et al., 1994; Funk et al., 2002; Sharples et al., 2005). El valor medio de la altitud y la pendiente se calculó con el comando de vecindad del SIG GRASS (neighbourhood operator), que genera un nuevo mapa raster en el cual se le asigna a cada celda el valor medio de los valores del área circular vecina, definida por los diferentes radios. Para estas variables medias se buscó un radio eficaz alrededor de los puntos de observación para el cual el ajuste del modelo multivariante fuera máximo (Daly et al., 1994; Kyriakidis et al., 2001; Guan et al., 2005). Finalmente, también se incluyeron en los modelos multivariantes de la precipitación los valores de radiación real (medida por los Satélites Meteosat), que es inversamente proporcional a la cobertura nubosa.

En los modelos multivariantes de la temperatura se incluyó la altitud, que suele ser la variable independiente con mayor peso (Daly, 2006). Los efectos de la continentalidad se analizaron con dos algoritmos de distancia a la costa (Agnew y Palutikof, 2000; Bustamante, 2003; Ninyerola et al., 2007b). El más sencillo es la distancia lineal, al que se sumó la distancia orográfica. Esta se calcula modificando la distancia lineal mediante un factor orográfico (en nuestro caso la altitud). Cada celda del mapa de distancia orográfica representa el coste acumulado de moverse a esa celda desde la línea de costa utilizando como superficie de coste la altitud (Neteler y Mitasova, 2004). Para incluir el efecto de las inversiones térmicas se calculó la diferencia entre la altitud puntual de cada estación y la altitud media para diferentes radios (los valores negativos indican zonas deprimidas, más proclives a las inversiones térmicas) (Perry y Hollis, 2005). Finalmente, los tres valores de radiación se incluyeron en los modelos separadamente para comprobar cuál de ellos daba mejores resultados.

\section{Interpolación de los residuos de las regresiones}

Los residuos de las regresiones (valor observado - valor de la regresión), que representan la variabilidad espacial en la variable no recogida por el modelo determinista de las funciones de regresión (Hengl, 2009), fueron interpolados 
por diferentes métodos. Después de varios ensayos previos se optó por la interpolación con métodos geostadísticos (Krigeado), al recoger este método la estructura de la correlación espacial (Hengl, 2009) y dar como resultado unos mapas con menos cráteres y picos (menos ruido). En este método de interpolación, los valores de los puntos estimados son medias ponderadas de los valores de los puntos existentes y los factores de ponderación se determinan a partir del semivariograma, de tal forma que se minimiza el error de cada punto calculado (Cressie, 1993). Se utilizó la opción de Krigeado ordinario, en la cual se especifica una distancia límite y un máximo y mínimo de puntos sobre los que calcular los factores de ponderación (ITC, 2001).

El variograma experimental utilizado en el Krigeado representa a la semivarianza $(\hat{\gamma})$ frente a la distancia $(\mathrm{h})$ entre los puntos observados, y se estimó mediante la fórmula:

$$
\hat{\gamma}=\sum\left(z_{i}-z_{i+h}\right)^{2} / 2 n
$$

Donde $z_{\mathrm{i}}$ es el valor del punto I, $z_{i+h}$ el valor de un punto a una distancia $\mathrm{h}$ del punto I y $n$ el número de pares de puntos dentro de una clase de distancia. Los modelos de variogramas se ajustaron al variograma experimental mediante la variación de los parámetros de efecto pepita, meseta y alcance.

\section{Validación de los modelos}

Los valores climáticos observados $\left(O_{i}\right)$ en las estaciones control (el $30 \%$ del total) se compararon con los valores estimados con los modelos $\left(E_{i}\right)$ por medio de los siguientes parámetros estadísticos: Coeficiente de determinación $\left(\mathrm{R}^{2}\right)$, Error Absoluto Medio (MAE, siglas del inglés «Mean Absolute Error»), Error Medio Cuadrático (RMSE, siglas de «Root Mean Square Error»), Error Medio (ME, siglas de «Mean Error»), ordenada en el origen ( $a$ ) y pendiente (b) de la regresión $E_{i}=a+b O_{i}$.

El R ${ }^{2}$, usado frecuentemente para analizar el ajuste de un modelo, puede ser un parámetro sesgado (Tveito, 2008), por lo que es importante utilizar también medidas de diferencia entre valores observados y estimados, como el MAE y el RMSE (Willmott, 1982). La ordenada en el origen (a) y la pendiente (b) de la regresión entre valores observados y estimados informan sobre la covarianza lineal entre ambos. 


\section{Resultados}

\section{Modelos de regresión multivariante}

La figura 6 muestra el $\mathrm{R}^{2}$ de las regresiones multivariantes de la precipitación media mensual para diferentes radios de las dos variables topográficas medias $\left(\mathrm{PEN}_{\mathrm{x}} \mathrm{y} \mathrm{ALT}_{\mathrm{x}}\right)$. Los mejores ajustes se obtuvieron con variables medias para radios de 30 y $15 \mathrm{~km}$. En los meses estivales (junio, julio y agosto) hubo pocas variaciones en el $\mathrm{R}^{2}$ con el radio. Para los modelos de temperatura la variable ALT-ALT $_{x}$ se mostró significativa para diferentes radios dependiendo de los meses ( $8 \mathrm{~km}$ para los meses de marzo y junio, $15 \mathrm{~km}$ para julio, agosto y septiembre y $30 \mathrm{~km}$ para octubre y noviembre).

\section{FIGURA 6}

\section{VALORES DEL R2 DE LAS REGRESIONES DE LA PRECIPITACIÓN}

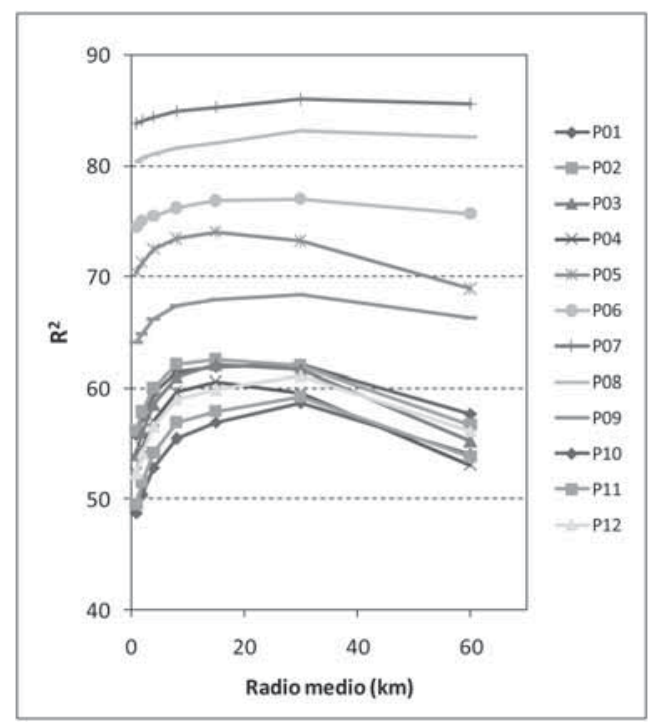

El ajuste de los modelos de regresión multivariante, en orden de complejidad creciente, se presenta en la figura 7. Los modelos con la altitud, longitud y latitud no resultaron muy buenos para la precipitación, excepto en los meses estivales. La adición de otras variables mejoró significativamente los modelos, particularmente la pendiente y altitud medias. Aunque la temperatura muestra un fuerte descenso con la elevación, esta pauta es más evidente en los meses no estivales (figura 7). El $\mathrm{R}^{2}$ para regresiones temperatura-altitud es menor 
de $50 \%$ para los meses de mayo a septiembre, con el valor más bajo en julio $(9,2 \%)$. Cuando se añaden otras variables el modelo mejora, particularmente en los meses estivales y los más cercanos a éstos.

FIGURA 7

AJUSTE DE LOS MODELOS DE REGRESIÓN MULTIVARIANTE
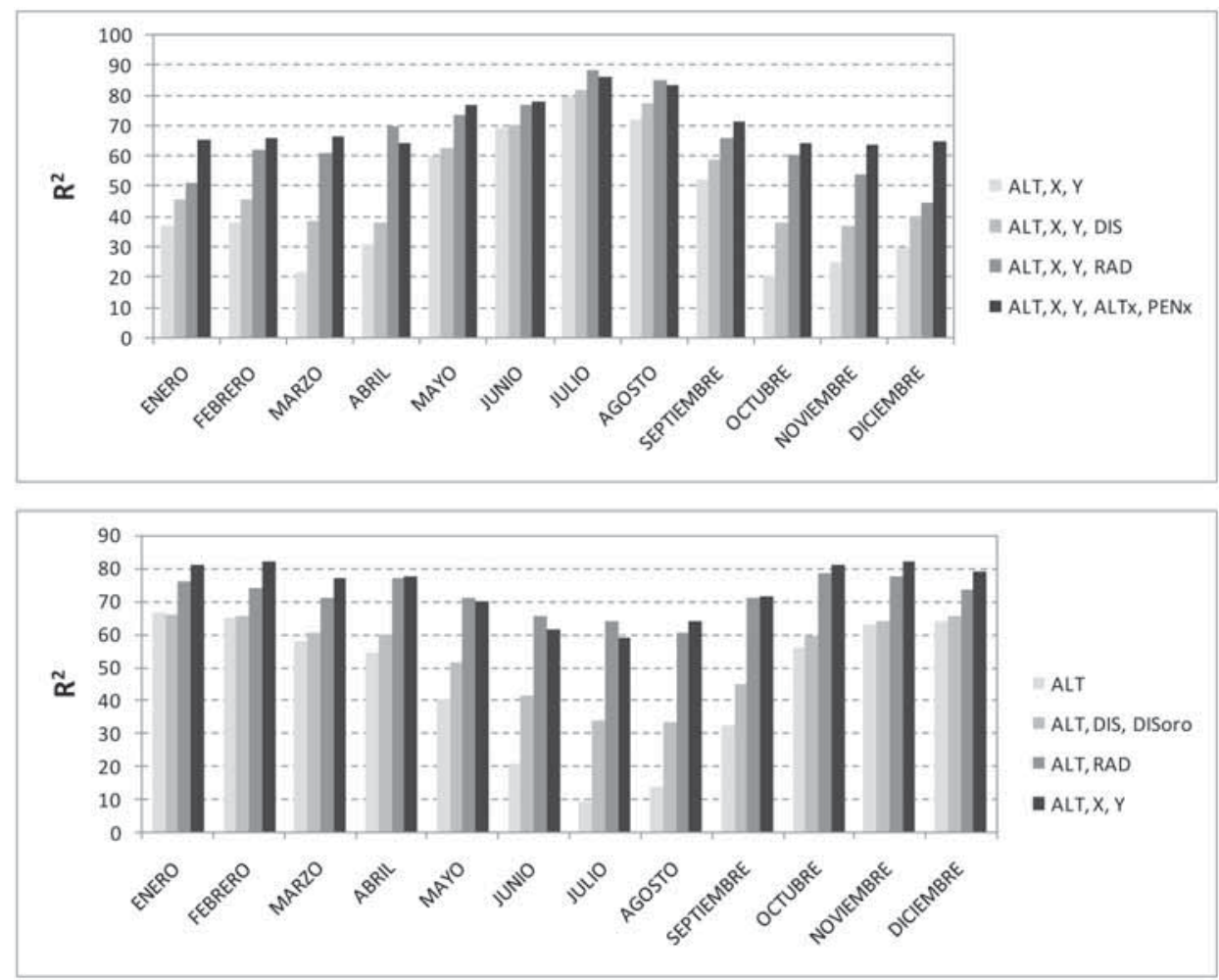

La influencia de la radiación en la temperatura dependió del conjunto de datos utilizados. La mayor correlación entre la temperatura y la radiación se obtuvo para la radiación real (datos del Meteosat) y la corregida (tabla 2). Los meses estivales mostraron las mejores correlaciones con la radiación. También se observaron diferencias en la influencia de los distintos tipos de radiación en los modelos multivariantes: la radiación potencial no fue estadísticamente significativa para las regresiones multivariantes de la temperatura en ningún mes del año, mientras que los otros dos tipos de radiación resultaron significativos en 8 meses. 
TABLA 2

CORRELACIÓN ENTRE LA RADIACIÓN Y LA TEMPERATURA

\begin{tabular}{l|c|c|c|c|c|c|c|c|c|c|c|c}
\hline & $\mathrm{T}_{1}$ & $\mathrm{~T}_{2}$ & $\mathrm{~T}_{3}$ & $\mathrm{~T}_{4}$ & $\mathrm{~T}_{5}$ & $\mathrm{~T}_{6}$ & $\mathrm{~T}_{7}$ & $\mathrm{~T}_{8}$ & $\mathrm{~T}_{9}$ & $\mathrm{~T}_{10}$ & $\mathrm{~T}_{11}$ & $\mathrm{~T}_{12}$ \\
\hline $\mathrm{RAD}_{\text {potencial }}$ & 0,284 & 0,271 & 0,238 & 0,181 & 0,041 & 0,006 & 0,145 & 0,275 & 0,314 & 0,300 & 0,308 & 0,289 \\
\hline $\mathrm{RAD}_{\text {real }}$ & 0,527 & 0,462 & 0,481 & 0,660 & 0,688 & 0,669 & 0,708 & 0,675 & 0,670 & 0,637 & 0,567 & 0,505 \\
\hline $\mathrm{RAD}_{\text {corregida }}$ & 0,444 & 0,404 & 0,439 & 0,645 & 0,687 & 0,683 & 0,717 & 0,667 & 0,617 & 0,558 & 0,482 & 0,414 \\
\hline
\end{tabular}

Las regresiones multivariantes utilizadas finalmente en los modelos mostraron un mejor ajuste para la temperatura (tabla 3). La importancia relativa de las variables independientes, analizada con los coeficientes estandarizados o coeficientes beta, se pueden ver en la tabla 4 .

Los variogramas utilizados para la interpolación de los residuos de las regresiones mediante Kriging (figura 8) presentaron variaciones entre meses y variables, con un buen ajuste $\left(R^{2}\right)$ entre el variograma experimental y el variograma modelo (tablas 5 y 6). Un ejemplo de los mapas de residuos de temperatura y precipitación del mes de enero, elaborados mediante kriging, se puede ver en la figura 9.

TABLA 3

DATOS DE LAS REGRESIONES MULTIVARIANTES

\begin{tabular}{|c|c|c|c|c|c|c|c|}
\hline & $\mathbf{R}^{2}$ & MAE & MEDIA & & $\mathbf{R}^{2}$ & MAE & MEDIA \\
\hline Ln-P & 71,2 & 0,25 & 4,15 & $\mathrm{~T}_{1}$ & 83,4 & 0,86 & 6,71 \\
\hline Ln- $\mathrm{P}_{2}$ & 72,9 & 0,23 & 4,04 & $\mathrm{~T}_{2}$ & 83,3 & 0,82 & 7,97 \\
\hline $\mathrm{Ln}-\mathrm{P}_{3}$ & 73,4 & 0,2 & 3,90 & $\mathrm{~T}_{3}$ & 79,4 & 0,89 & 10,19 \\
\hline Ln- $\mathrm{P}_{4}$ & 76,5 & 0,16 & 4,08 & $\mathrm{~T}_{4}$ & 83,2 & 0,81 & 11,95 \\
\hline Ln- $\mathrm{P}_{5}$ & 79,1 & 0,16 & 4,00 & $\mathrm{~T}_{5}$ & 79,8 & 0,87 & 15,53 \\
\hline Rc- $P_{6}$ & 80,1 & 0,54 & 6,02 & $\mathrm{~T}_{6}$ & 80,0 & 0,95 & 19,71 \\
\hline Rc- $P_{7}$ & 89,4 & 0,46 & 3,95 & $\mathrm{~T}_{7}$ & 80,3 & 1,04 & 23,02 \\
\hline Rc- $P_{8}$ & 86,3 & 0,57 & 4,40 & $\mathrm{~T}_{8}$ & 80,7 & 1,02 & 22,97 \\
\hline $\mathrm{Ln}-\mathrm{P}_{9}$ & 72,6 & 0,2 & 3,67 & $\mathrm{~T}_{9}$ & 81,3 & 0,90 & 19,85 \\
\hline Ln-P ${ }_{10}$ & 71,2 & 0,17 & 4,20 & $\mathrm{~T}_{10}$ & 83,8 & 0,81 & 15,1 \\
\hline Ln- ${ }_{11}$ & 71,2 & 0,2 & 4,29 & $\mathrm{~T}_{11}$ & 84,1 & 0,82 & 10,27 \\
\hline Ln-P ${ }_{12}$ & 70,8 & 0,25 & 4,25 & $\mathrm{~T}_{12}$ & 82,5 & 0,90 & 7,39 \\
\hline
\end{tabular}

Coeficientes de determinación (R2) y errores absolutos medios (MAE) de las regresiones multivariantes de la precipitación transformada (como logaritmo natural, Ln, o raíz cuadrada, Rc) y la temperatura, frente a distintas variables independientes. Se indica también el valor medio de cada variable.

Estudios Geográficos, Vol. LXXVIII, 283, pp. 493-522, julio-diciembre 2017

ISSN: 0014-1496, eISSN: 1988-8546, doi: 10.3989/estgeogr.201717 
TABLA 4

COEFICIENTES BETA DE LAS REGRESIONES.

\begin{tabular}{|c|c|c|c|c|c|c|c|}
\hline & \multicolumn{7}{|c|}{ Precipitación mensual } \\
\hline & $X$ & $\mathrm{Y}$ & ALT & $\operatorname{ALT}_{x}$ & $\operatorname{PEN}_{x}$ & DIS & $\mathrm{RAD}_{\text {real }}$ \\
\hline $\log -P_{\text {enero }}$ & $-0,44$ & $-0,81$ & 0,35 & $-0,75$ & 0,62 & 0,09 & $-1,02$ \\
\hline Log-P $\mathrm{P}_{\text {febrero }}$ & $-0,42$ & $-0,58$ & 0,35 & $-0,68$ & 0,52 & 0,20 & $-0,79$ \\
\hline Log-P ${ }_{\text {marzo }}$ & $-0,29$ & $-0,42$ & 0,44 & $-0,85$ & 0,57 & 0,19 & $-0,77$ \\
\hline Log-P ${ }_{\text {abril }}$ & $-0,03$ & $-0,32$ & 0,36 & $-0,76$ & 0,38 & 0,23 & $-0,99$ \\
\hline Log-P ${ }_{\text {mayo }}$ & $-0,04$ & 0,35 & 0,42 & $-0,56$ & 0,39 & 0,20 & $-0,42$ \\
\hline Rc- $P_{\text {junio }}$ & 0,06 & 0,44 & 0,26 & $-0,19$ & 0,27 & 0,21 & $-0,35$ \\
\hline Rc-P ${ }_{\text {julio }}$ & 0,12 & 0,51 & 0,11 & & 0,13 & 0,10 & $-0,42$ \\
\hline Rc-P ${ }_{\text {agosto }}$ & 0,28 & 0,38 & 0,13 & $-0,21$ & 0,19 & 0,05 & $-0,48$ \\
\hline Log-P ${ }_{\text {septiembre }}$ & 0,15 & 0,41 & 0,38 & $-0,72$ & 0,43 & 0,12 & $-0,35$ \\
\hline Log-P ${ }_{\text {octubre }}$ & $-0,08$ & $-0,61$ & 0,36 & $-0,88$ & 0,51 & 0,07 & $-1,08$ \\
\hline Log-P $\mathrm{P}_{\text {noviembre }}$ & $-0,27$ & $-0,85$ & 0,36 & $-0,86$ & 0,63 & 0,14 & $-1,13$ \\
\hline \multirow[t]{3}{*}{$\log -\mathrm{P}_{\text {diciembre }}$} & $-0,31$ & $-0,85$ & 0,38 & $-0,82$ & 0,67 & 0,05 & $-1,03$ \\
\hline & \multicolumn{7}{|c|}{ Temperatura media mensual } \\
\hline & $\mathrm{X}$ & $\mathrm{Y}$ & ALT & DIS & DIS $_{\text {oro }}$ & ALT-ALT $_{x}$ & $\mathrm{RAD}_{\text {corr }}$ \\
\hline $\mathrm{T}_{\text {enero }}$ & $-0,10$ & $-0,41$ & $-0,56$ & $-0,08$ & $-0,15$ & & \\
\hline $\mathrm{T}_{\text {febrero }}$ & $-0,07$ & $-0,42$ & $-0,62$ & 0,06 & $-0,19$ & & \\
\hline $\mathrm{T}_{\text {marzo }}$ & & $-0,41$ & $-0,64$ & 0,22 & $-0,26$ & & 0,06 \\
\hline $\mathrm{T}_{\mathrm{abril}}$ & & $-0,30$ & $-0,58$ & 0,28 & $-0,28$ & $-0,06$ & 0,25 \\
\hline $\mathrm{T}_{\text {mayo }}$ & 0,14 & $-0,32$ & $-0,55$ & 0,40 & $-0,30$ & $-0,06$ & 0,27 \\
\hline $\mathrm{T}_{\text {junio }}$ & 0,25 & $-0,35$ & $-0,59$ & 0,55 & $-0,28$ & & 0,31 \\
\hline $\mathrm{T}_{\text {julio }}$ & 0,29 & $-0,41$ & $-0,49$ & 0,58 & $-0,26$ & & 0,30 \\
\hline $\mathrm{T}_{\text {agosto }}$ & 0,34 & $-0,45$ & $-0,54$ & 0,55 & $-0,25$ & 0,04 & 0,24 \\
\hline $\mathrm{T}_{\text {septiembre }}$ & 0,20 & $-0,51$ & $-0,66$ & 0,48 & $-0,25$ & 0,06 & 0,11 \\
\hline $\mathrm{T}_{\text {octubre }}$ & 0,09 & $-0,45$ & $-0,70$ & 0,27 & $-0,24$ & 0,05 & 0,06 \\
\hline $\mathrm{T}_{\text {noviembre }}$ & $-0,03$ & $-0,45$ & $-0,60$ & & $-0,17$ & 0,03 & \\
\hline $\mathrm{T}_{\text {diciembre }}$ & $-0,10$ & $-0,40$ & $-0,52$ & $-0,13$ & $-0,15$ & & \\
\hline
\end{tabular}

Coeficientes beta estandarizados de las variable independientes de los modelos de regresión múltiple de la precipitación transformada y la temperaturas. 
FIGURA 8

VARIOGRAMAS UTILIZADOS EN LA INTERPOLACIÓN DE LOS RESIDUOS

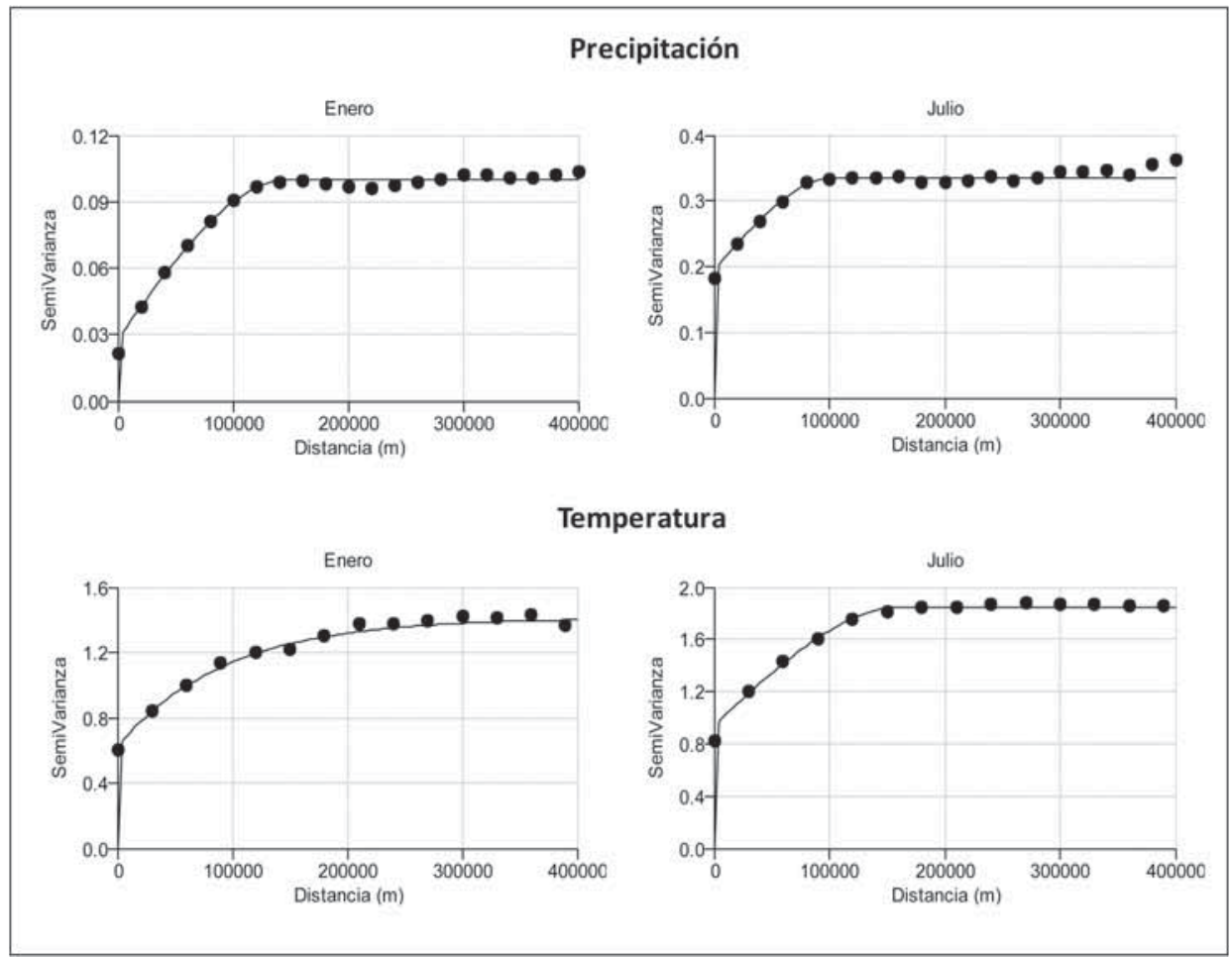

FIGURA 9

MAPAS DE DE LOS RESIDUOS DE LA PRECIPITACIÓN Y TEMPERATURA
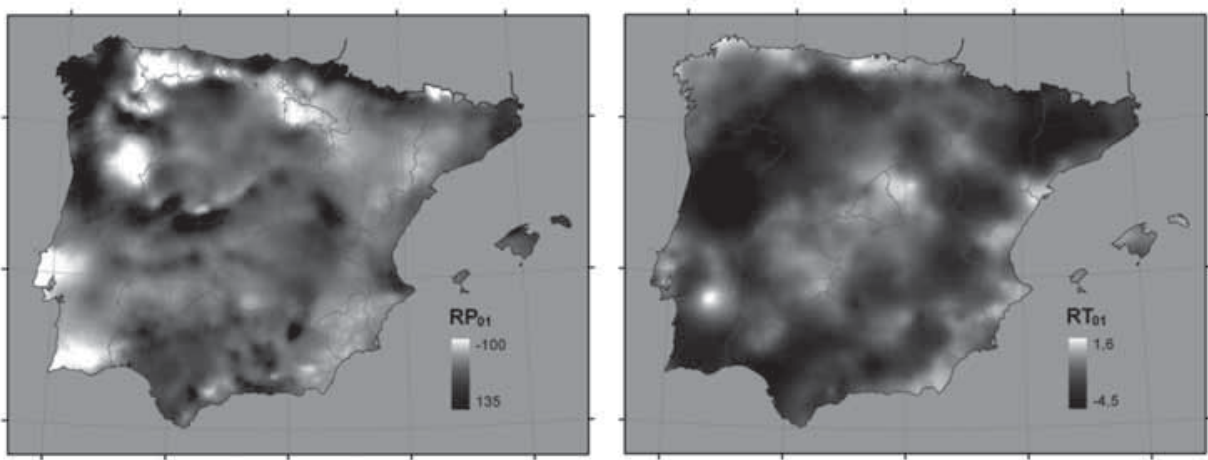

Estudios Geográficos, Vol. LXXVIII, 283, pp. 493-522, julio-diciembre 2017 ISSN: 0014-1496, eISSN: 1988-8546, doi: 10.3989/estgeogr.201717 
TABLA 5

PARÁMETROS DE LOS VARIOGRAMAS DE LA PRECIPITACIÓN

\begin{tabular}{|c|c|c|c|c|c|c|}
\hline & \multirow[b]{2}{*}{ Modelo } & \multirow[b]{2}{*}{$\mathrm{R}^{2}(\%)$} & \multicolumn{4}{|c|}{ Parámetros } \\
\hline & & & Efecto pepita & Meseta & $\begin{array}{c}\text { Efecto pepita/ } \\
\text { Meseta }(\%)\end{array}$ & Rango (m) \\
\hline Log- $\mathrm{P}_{\text {enero }}$ & Esférico & 0,99 & 0,028 & 0,100 & 28 & 148000 \\
\hline $\log -P_{\text {febrero }}$ & Esférico & 0,99 & 0,029 & 0,092 & 32 & 152000 \\
\hline $\log -\mathrm{P}_{\text {marzo }}$ & Esférico & 0,99 & 0,029 & 0,066 & 44 & 155000 \\
\hline Log-P abril & Esférico & 0,98 & 0,019 & 0,040 & 48 & 160000 \\
\hline Log- $\mathrm{P}_{\text {mayo }}$ & Esférico & 0,92 & 0,019 & 0,040 & 48 & 150000 \\
\hline Rc- $P_{\text {junio }}$ & Exponencial & 0,93 & 0,200 & 0,460 & 43 & 88000 \\
\hline Rc-P ${ }_{\text {julio }}$ & Esférico & 0,91 & 0,195 & 0,335 & 58 & 105000 \\
\hline Rc-P $\mathrm{agosto}_{\text {ags }}$ & Exponencial & 0,97 & 0,148 & 0,510 & 29 & 60000 \\
\hline Log-P $\mathrm{P}_{\text {septiembre }}$ & Exponencial & 0,96 & 0,026 & 0,068 & 38 & 112000 \\
\hline Log-P octubre & Exponencial & 0,95 & 0,016 & 0,056 & 28 & 80000 \\
\hline Log- $\mathrm{P}_{\text {noviembre }}$ & Esférico & 0,99 & 0,024 & 0,067 & 36 & 162000 \\
\hline Log-P $P_{\text {diciembre }}$ & Esférico & 0,98 & 0,026 & 0,095 & 27 & 145000 \\
\hline
\end{tabular}

Parámetros de los semivariogramas de los residuos de las regresiones multivariantes de las precipitaciones.

TABLA 6

PARÁMETROS DE LOS VARIOGRAMAS DE LA TEMPERATURA

\begin{tabular}{l|c|c|c|c|c|c}
\hline & \multirow{2}{*}{ Modelo } & \multirow{2}{*}{$\mathrm{R}^{2}(\%)$} & \multicolumn{4}{|c}{ Parámetros } \\
\cline { 5 - 7 } & & & Efecto pepita & Meseta & $\begin{array}{c}\text { Efecto pepita/ } \\
\text { Meseta (\%) }\end{array}$ & Rango (m) \\
\hline $\mathrm{T}_{\text {enero }}$ & Exponencial & 98,1 & 0,620 & 1,410 & 44 & 93000 \\
\hline $\mathrm{T}_{\text {febrero }}$ & Exponencial & 98,3 & 0,670 & 1,200 & 56 & 76000 \\
\hline $\mathrm{T}_{\text {marzo }}$ & Exponencial & 96,5 & 0,830 & 1,400 & 59 & 67000 \\
\hline $\mathrm{T}_{\text {abril }}$ & Exponencial & 97,6 & 0,710 & 1,210 & 59 & 70100 \\
\hline $\mathrm{T}_{\text {mayo }}$ & Exponencial & 98,3 & 0,840 & 1,350 & 62 & 72000 \\
\hline $\mathrm{T}_{\text {junio }}$ & Esférico & 98,5 & 0,930 & 1,570 & 59 & 165000 \\
\hline $\mathrm{T}_{\text {julio }}$ & Esférico & 99,5 & 0,940 & 1,850 & 51 & 165000 \\
\hline $\mathrm{T}_{\text {agosto }}$ & Esférico & 99,8 & 0,980 & 1,770 & 55 & 170000 \\
\hline $\mathrm{T}_{\text {septiembre }}$ & Esférico & 98,4 & 0,880 & 1,470 & 60 & 167000 \\
\hline $\mathrm{T}_{\text {octubre }}$ & Exponencial & 97,0 & 0,680 & 1,200 & 57 & 70000 \\
\hline $\mathrm{T}_{\text {noviembre }}$ & Exponencial & 96,0 & 0,620 & 1,270 & 49 & 77000 \\
\hline $\mathrm{T}_{\text {diciembre }}$ & Exponencial & 96,5 & 0,630 & 1,440 & 44 & 86000 \\
\hline
\end{tabular}

Parámetros de los semivariogramas de los residuos de las regresiones multivariantes de las temperaturas. 


\section{Validación de los modelos}

Al comparar los modelos de cálculo de las variables usando solamente la regresión multivariante o usando ésta más la interpolación de los residuos, se observan claras diferencias entre parámetros climáticos y meses. En general, para la precipitación, el ajuste entre datos calculados y observados aumentó cuando se utilizó la interpolación de los residuos, principalmente en los meses no estivales (tabla 7). Sin embargo, para la temperatura, las diferencias entre ambos métodos fueron menores (tabla 8).

Por otro lado, los errores de las estimaciones no son iguales para todo el rango de valores de la variable, como se muestra en la figura 10, que representa las regresiones entre valores observados y estimados de precipitación total anual y la temperatura media anual. Para la precipitación, los puntos se encuentran más dispersos en los valores más altos mientras que para la temperatura, la distribución de los puntos es más regular a lo largo de toda la línea de regresión.

La comparación de los datos de precipitación y temperatura con los de los 60 observatorios principales (figura 11) para los tres últimos periodos climatológicos referenciales muestra un buen grado de correlación, así como un error que varía en función del periodo (tablas 10 y 11).

TABLA 7

PARÁMETROS DE LA VALIDACIÓN DE LOS MODELOS DE PRECIPITACIÓN.

\begin{tabular}{|c|c|c|c|c|c|c|c|c|}
\hline & \multicolumn{8}{|c|}{ Métodos } \\
\hline & \multicolumn{2}{|c|}{ Regresión } & \multicolumn{6}{|c|}{ Regresión + interpolación de residuos } \\
\hline & $\mathrm{R}^{2}$ & MAE & $\mathrm{R}^{2}$ & MAE & RMSE & ME & $\mathrm{a}$ & $\mathrm{b}$ \\
\hline $\mathrm{P}_{\text {enero }}$ & 69,5 & 14,4 & 90,0 & 8,7 & 15,0 & 1,5 & 7,7 & 0,9 \\
\hline $\mathrm{P}_{\text {febrero }}$ & 69,7 & 12,8 & 89,1 & 8,7 & 13,4 & $-2,4$ & 7,9 & 0,9 \\
\hline $\mathrm{P}_{\text {marzo }}$ & 71,6 & 9,8 & 87,7 & 7,0 & 12,0 & 1,6 & 7,7 & 0,8 \\
\hline $\mathrm{P}_{\text {abril }}$ & 78,1 & 8,7 & 90,5 & 6,3 & 10,1 & 0,8 & 6,9 & 0,9 \\
\hline $\mathrm{P}_{\text {mayo }}$ & 78,6 & 8,3 & 91,3 & 5,6 & 9,0 & 0,9 & 6,7 & 0,9 \\
\hline $\mathrm{P}_{\text {junio }}$ & 77,0 & 6,2 & 90,3 & 4,2 & 6,5 & 0,4 & 4,2 & 0,9 \\
\hline $\mathrm{P}_{\text {julio }}$ & 86,3 & 3,9 & 94,2 & 2,7 & 4,4 & 0,3 & 1,3 & 0,9 \\
\hline $\mathrm{P}_{\text {agosto }}$ & 83,4 & 5,2 & 94,8 & 3,2 & 5,1 & 0,4 & 0,7 & 0,9 \\
\hline $\mathrm{P}_{\text {septiembre }}$ & 69,3 & 7,6 & 90,0 & 5,1 & 7,7 & 0,9 & 5,0 & 0,9 \\
\hline $\mathrm{P}_{\text {octubre }}$ & 73,1 & 10,6 & 90,7 & 7,1 & 11,1 & 1,1 & 7,9 & 0,9 \\
\hline $\mathrm{P}_{\text {noviembre }}$ & 71,9 & 13,6 & 90,1 & 8,7 & 13,9 & 1,0 & 8,8 & 0,9 \\
\hline $\mathrm{P}_{\text {diciembre }}$ & 70,0 & 16,0 & 89,1 & 9,8 & 16,4 & 0,9 & 6,3 & 0,9 \\
\hline $\mathrm{P}_{\text {anual }}$ & 76,8 & 96,9 & 92,5 & 59,4 & 98,1 & 7,4 & 59,4 & 0,9 \\
\hline
\end{tabular}

Estudios Geográficos, Vol. LXXVIII, 283, pp. 493-522, julio-diciembre 2017

ISSN: 0014-1496, eISSN: 1988-8546, doi: 10.3989/estgeogr.201717 
TABLA 8

PARÁMETROS DE LA VALIDACIÓN DE LOS MODELOS DE TEMPERATURA.

\begin{tabular}{|c|c|c|c|c|c|c|c|c|}
\hline & \multicolumn{8}{|c|}{ Métodos } \\
\hline & \multicolumn{2}{|c|}{ Regresión } & \multicolumn{6}{|c|}{ Regresión + interpolación de residuos } \\
\hline & $\mathrm{R}^{2}$ & MAE & $\mathrm{R}^{2}$ & MAE & RMSE & ME & a & $\mathrm{b}$ \\
\hline $\mathrm{T}_{\text {enero }}$ & 86,3 & 0,81 & 90,6 & 0,68 & 0,88 & $-0,03$ & 0,06 & 0,99 \\
\hline $\mathrm{T}_{\text {febrero }}$ & 86,7 & 0,78 & 89,7 & 0,69 & 0,90 & $-0,04$ & 0,05 & 0,99 \\
\hline $\mathrm{T}_{\text {marzo }}$ & 83,8 & 0,82 & 86,3 & 0,75 & 0,95 & 0,03 & 0,12 & 0,99 \\
\hline $\mathrm{T}_{\text {abril }}$ & 86,5 & 0,74 & 88,0 & 0,68 & 0,91 & 0,08 & 0,43 & 0,97 \\
\hline $\mathrm{T}_{\text {mayo }}$ & 84,1 & 0,79 & 86,7 & 0,69 & 0,92 & 0,06 & 0,31 & 0,98 \\
\hline $\mathrm{T}_{\text {junio }}$ & 82,3 & 0,90 & 86,5 & 0,76 & 1,00 & 0,05 & 0,30 & 0,99 \\
\hline $\mathrm{T}_{\text {julio }}$ & 83,0 & 0,98 & 88,1 & 0,81 & 1,04 & 0,09 & 0,09 & 1,00 \\
\hline $\mathrm{T}_{\text {agosto }}$ & 83,2 & 0,96 & 87,9 & 0,82 & 1,04 & 0,09 & 0,12 & 1,00 \\
\hline $\mathrm{T}_{\text {septiembre }}$ & 85,6 & 0,82 & 89,2 & 0,71 & 0,90 & 0,05 & 0,03 & 1,00 \\
\hline $\mathrm{T}_{\text {octubre }}$ & 88,5 & 0,72 & 90,5 & 0,64 & 0,83 & 0,01 & 0,08 & 1,00 \\
\hline $\mathrm{T}_{\text {noviembre }}$ & 88,2 & 0,76 & 91,3 & 0,65 & 0,86 & $-0,03$ & $-0,01$ & 1,00 \\
\hline $\mathrm{T}_{\text {diciembre }}$ & 85,7 & 0,73 & 90,3 & 0,69 & 0,89 & $-0,04$ & 0,05 & 0,99 \\
\hline $\mathrm{T}_{\text {media anual }}$ & 87,0 & 0,73 & 89,9 & 0,63 & 1,19 & $-0,02$ & 0,09 & 1,00 \\
\hline
\end{tabular}

FIGURA 10

REGRESIONES ENTRE LOS VALORES OBSERVADOS Y CALCULADOS
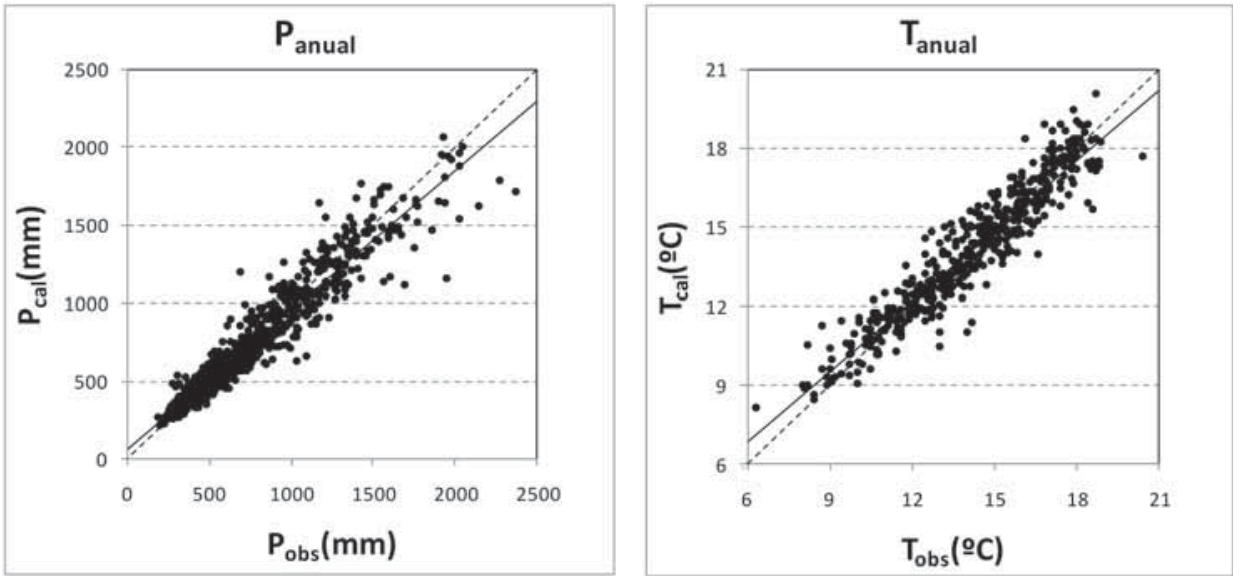

Estudios Geográficos, Vol. LXXVIII, 283, pp. 493-522, julio-diciembre 2017 ISSN: 0014-1496, eISSN: 1988-8546, doi: 10.3989/estgeogr.201717 
FIGURA 11

\section{OBSERVATORIOS METEOROLÓGICOS PRINCIPALES}

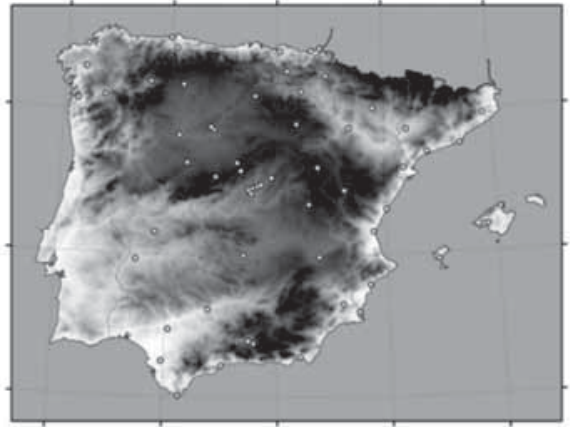

TABLA 9

PRECIPITACIÓN Y TEMPERATURA DE LOS OBSERVATORIOS PRINCIPALES

\begin{tabular}{l|c|c|c|l|c|c|c}
\hline & $1961-1990$ & $1971-2000$ & $1981-2010$ & & $1961-1990$ & $1971-2000$ & $1981-2010$ \\
\hline $\mathrm{P}_{\text {enero }}$ & 66,8 & 61,4 & 56,2 & $\mathrm{~T}_{\text {enero }}$ & 7,1 & 7,2 & 7,4 \\
\hline $\mathrm{P}_{\text {abril }}$ & 61,9 & 59,0 & 56,8 & $\mathrm{~T}_{\text {abril }}$ & 11,9 & 12,1 & 12,7 \\
\hline $\mathrm{P}_{\text {julio }}$ & 20,7 & 19,9 & 18,1 & $\mathrm{~T}_{\text {julio }}$ & 22,6 & 22,8 & 23,4 \\
\hline $\mathrm{P}_{\text {octubre }}$ & 67,2 & 69,1 & 74,7 & $\mathrm{~T}_{\text {octubre }}$ & 15,3 & 15,4 & 15,9 \\
\hline $\mathrm{P}_{\text {anual }}$ & 638,7 & 610,9 & 601,4 & $\mathrm{~T}_{\text {media anual }}$ & 14,2 & 14,5 & 14,9 \\
\hline
\end{tabular}

Valores de precipitación total y temperatura media de los Observatorios Principales de la red española $(n=60)$, para los tres últimos periodos climatológicos referenciales. Meses centrales de cada estación y valor anual.

TABLA 10

COMPARACIÓN DE LA PRECIPITACIÓN ENTRE EL MODELO Y LOS OBSERVATORIOS PRINCIPALES

\begin{tabular}{l|c|c|c|c|c|c}
\hline \multirow{2}{*}{} & \multicolumn{2}{|c|}{$1961-1990$} & \multicolumn{2}{c|}{$1971-2000$} & \multicolumn{2}{c}{$1981-2010$} \\
\cline { 2 - 7 } & $\mathrm{R}^{2}$ & $\mathrm{ME}$ & $\mathrm{R}^{2}$ & $\mathrm{ME}$ & $\mathrm{R}^{2}$ & $\mathrm{ME}$ \\
\hline $\mathrm{P}_{\text {enero }}$ & 97,4 & 0,69 & 86,4 & $-4,76$ & 84,9 & $-9,91$ \\
\hline $\mathrm{P}_{\text {abril }}$ & 94,9 & 2,65 & 82,8 & $-0,25$ & 84,0 & $-2,48$ \\
\hline $\mathrm{P}_{\text {julio }}$ & 96,1 & 0,68 & 88,5 & 1,53 & 84,5 & $-1,09$ \\
\hline $\mathrm{P}_{\text {octubre }}$ & 93,0 & $-2,79$ & 85,5 & $-0,93$ & 83,8 & 4,64 \\
\hline $\mathrm{P}_{\text {anual }}$ & 95,9 & 6,16 & 86,5 & $-14,74$ & 87,5 & $-31,47$ \\
\hline
\end{tabular}

Coeficiente de determinación $\left(\mathrm{R}^{2}\right)$ y error medio $(\mathrm{ME})$, en $(\mathrm{mm})$, entre los datos de precipitación medidos los tres últimos periodos climatológicos referenciales y los calculados mediante el modelo climático para las Estaciones Principales de la red española $(\mathrm{n}=60)$. Meses centrales de cada estación y valor anual.

Estudios Geográficos, Vol. LXXVIII, 283, pp. 493-522, julio-diciembre 2017

ISSN: 0014-1496, eISSN: 1988-8546, doi: 10.3989/estgeogr.201717 
TABLA 11

COMPARACIÓN DE LA TEMPERATURA ENTRE EL MODELO Y LOS OBSERVATORIOS PRINCIPALES.

\begin{tabular}{l|c|c|c|c|c|c}
\hline \multirow{2}{*}{} & \multicolumn{2}{|c|}{$1961-1990$} & \multicolumn{2}{c|}{$1971-2000$} & \multicolumn{2}{c}{$1981-2010$} \\
\cline { 2 - 7 } & $\mathbf{R}^{2}$ & $\mathbf{M E}$ & $\mathbf{R}^{2}$ & $\mathbf{M E}$ & $\mathbf{R}^{2}$ & $\mathbf{M E}$ \\
\hline $\mathrm{T}_{\text {enero }}$ & 96,9 & $-0,08$ & 93,0 & $-0,01$ & 88,9 & 0,19 \\
\hline $\mathrm{T}_{\text {abril }}$ & 93,6 & $-0,39$ & 85,1 & $-0,18$ & 85,8 & 0,45 \\
\hline $\mathrm{T}_{\text {julio }}$ & 94,8 & $-0,48$ & 90,1 & $-0,24$ & 86,4 & 0,35 \\
\hline $\mathrm{T}_{\text {octubre }}$ & 96,4 & $-0,06$ & 89,7 & $-0,14$ & 90,2 & 0,40 \\
\hline $\mathrm{T}_{\text {media anual }}$ & 95,8 & $-0,31$ & 89,9 & $-0,07$ & 89,7 & 0,36 \\
\hline
\end{tabular}

Coeficiente de determinación $\left(\mathrm{R}^{2}\right)$ y error medio $(\mathrm{ME})$, en $\left({ }^{\circ} \mathrm{C}\right)$, entre los datos de temperatura medidos los tres últimos periodos climatológicos referenciales y los calculados mediante el modelo climático para las Estaciones Principales de la red española $(n=60)$. Meses centrales de cada estación y valor anual.

\section{Mapas de precipitación y temperatura}

En las figuras 12 y 13 se presentan mapas de precipitación y temperatura estimados de enero y julio. En estos mapas se pueden ver las tendencias conocidas de estas variables climáticas a lo largo de la península ibérica (localización de los sectores más lluviosos en el norte y noroeste, marcadas diferencias en la cantidad de precipitación, particularmente para el mes de julio, y diferencias en las temperaturas entre las zonas de mayor y menor altitud, zonas costeras y de interior, entre el norte y el sur de la península ibérica, etc.).

FIGURA 12

\section{MAPAS DE PRECIPITACIONES MENSUALES ESTIMADAS}
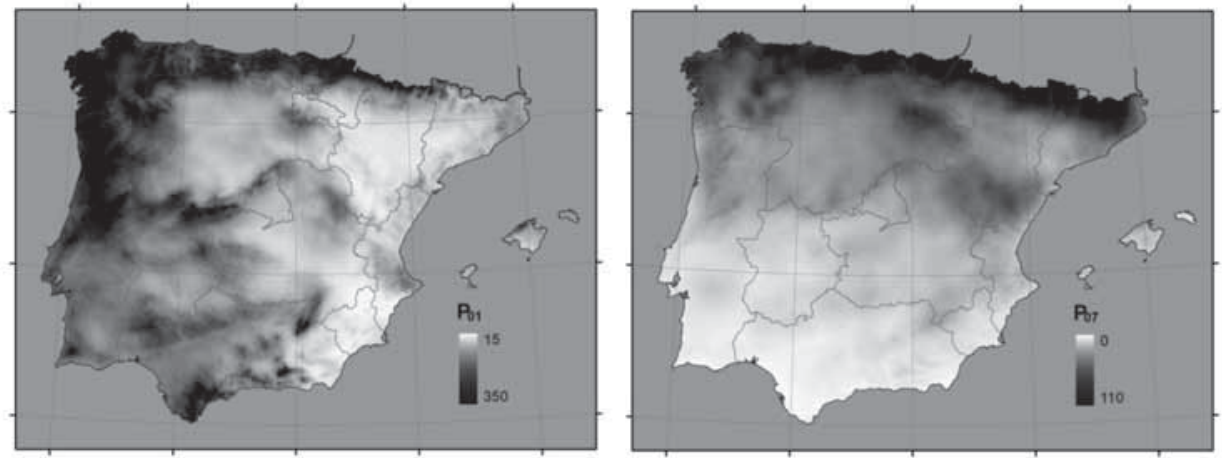

Estudios Geográficos, Vol. LXXVIII, 283, pp. 493-522, julio-diciembre 2017 ISSN: 0014-1496, eISSN: 1988-8546, doi: 10.3989/estgeogr.201717 
FIGURA 13

MAPAS DE TEMPERATURAS MEDIAS MENSUALES ESTIMADAS
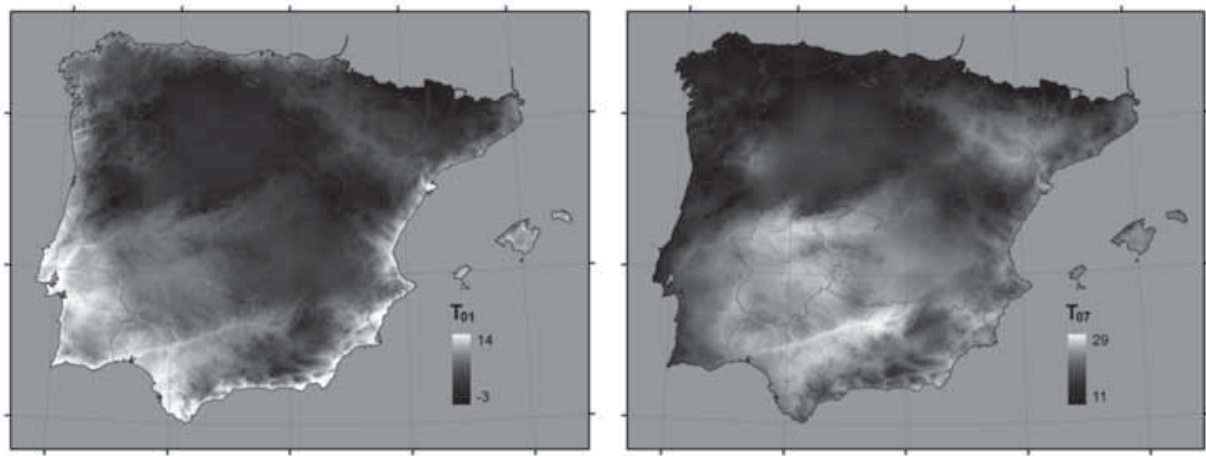

DisCUSIÓN

\section{Precipitación}

Los trabajos de interpolación de la precipitación mediante regresión multivariante han utilizado tradicionalmente la altitud y las coordenadas geográficas como variables independientes (Xin et al., 2007). El uso de variables topográficas medias mejora significativamente el ajuste de los modelos de regresión multivariante (Agnew y Palutikof, 2000; Vicente-Serrano et al., 2003), como se ha observado en el presente trabajo. Para algunos de los meses de más alta precipitación, el $\mathrm{R}^{2}$ de las regresiones aumenta más del $10 \%$ cuando se utilizan la altitud y pendiente medias (figura 7). Esto tiene que ver con la escala a la que se producen los fenómenos de precipitación. Así, los sistemas frontales tienen una extensión mucho mayor que las células convectivas estivales. Por eso, las precipitaciones de los meses de octubre a abril presentan mejores ajustes en el modelo multivariante para las variables topográficas medias de 15 o $30 \mathrm{~km}$, mientras en los meses estivales el modelo no mejora casi nada cuando se aumenta el radio de las variables (figura 6). La escala a la que se manifiestan los efectos de la topografía (radio de las variables topográficas medias) es similar a la observada en otros trabajos (Daly et al., 1994; Agnew y Palutikof, 2000; Vicente-Serrano et al., 2003; Guan et al., 2005).

En líneas generales la precipitación aumenta con la altitud (tabla 4), por el ascenso y enfriamiento consiguiente de los vientos húmedos cuando atraviesan obstáculos en el terreno de suficiente magnitud (Barry, 1992). Por la misma razón la precipitación también aumenta con la pendiente media, 
cuando los sistemas montañosos obstaculizan las masas de aire húmedo, hecho significativo en los relieves peninsulares. La altitud media, sin embargo, muestra un comportamiento contrario: una correlación negativa con la precipitación, con un mayor peso de los coeficientes durante los meses con precipitaciones asociadas a frentes (octubre a abril). No siempre es fácil entender las correlaciones en los modelos multivariantes, más cuando intervienen gran número de variables, aunque en este caso sí que podemos encontrar alguna explicación. El signo negativo de la altitud media en los modelos puede tener que ver con la configuración de la península ibérica como una extensa planicie volcada hacia el suroeste, interrumpida por varios sistemas montañosos. Considerando que la mayoría de las precipitaciones ciclonales vienen por el oeste, esta planicie actúa como superficie que se interpone y hace que las lluvias vayan descargando a su paso por ella y en los relieves intercalados. De esta forma, cuando se llega a las zonas más altas, las masas de aire ya han perdido parte de su humedad, lo que explicaría que una mayor altitud media se correlacionara negativamente con la precipitación en el modelo multivariante.

Las coordenadas geográficas han tenido un peso variable en el modelo dependiendo del mes del año, en función de la mayor o menor influencia de las lluvias procedentes de los distintos puntos cardinales (tabla 4). Por su parte, la radiación real (medida con los satélites Meteosat), mostró una fuerte correlación negativa con la precipitación por su relación inversa con la nubosidad, particularmente en los meses más lluviosos del año. En otros trabajos que han utilizado la radiación potencial, el modelo de precipitaciones no se ha visto mejorado, dada la poca relación que tiene esta variable con la precipitación (Ninyerola et al., 2000a; Vicente-Serrano et al., 2003; Ninyerola et al., 2007a). La distancia a la costa tiene un menor peso en el modelo debido al diferente comportamiento en cuanto a la precipitación de los mares que rodean a la península. El Mediterráneo influye más en las precipitaciones del final del verano y principios del otoño mientras que del Atlántico proceden la mayor parte de las precipitaciones ciclonales primaverales y otoñales (Capel, 2000). La inclusión por separado de las distancias a los diferentes mares tampoco mejoró el modelo.

La correlación espacial de los residuos de las precipitaciones viene dada por el buen ajuste de los residuos a los variogramas (tabla 5). El modelo multivariante no recoge por tanto toda la variabilidad de los datos de precipitación mensual. Esta desviación del modelo puede estar relacionada con particularidades climáticas regionales difíciles de detectar a mayor escala. Además, hay una variación espacial de los residuos de las regresiones que no puede explicar el variograma, dada por el efecto pepita, debido principalmente 
a la escala de muestreo, errores de medición y variación de la variable a pequeñas distancias (Cressie, 1993). Esta variación a pequeña escala es menor en la precipitación que en la temperatura ya que hay un valor más bajo en la relación entre el efecto pepita y la meseta (tablas 5 y 6). Esto puede estar influido también por la mayor densidad de estaciones de precipitación frente a las de temperatura.

El modelo de estimación de las precipitaciones mejora significativamente cuando se interpolan los residuos de las regresiones a lo largo de la zona de estudio (tabla 7), como se ha observado en otros estudios (Agnew y Palutikof, 2000; Kyriakidis et al., 2001; Brown y Comrie, 2002; Vicente-Serrano et al., 2003; Guan et al., 2005; Ninyerola et al., 2007a; Ninyerola et al., 2007b). Uno de los problemas de la interpolación de los residuos es la obtención de superficies irregulares, con cráteres y picos, lo cual puede tener su repercusión en los mapas finales de las variables climáticas (como sucede en alguno de los mapas precipitaciones y temperaturas del atlas climático de Ninyerola y colaboradores (http://www.opengis.uab.es/wms/iberia/mms/index.htm). Por tanto, la obtención de buenos ajustes estadísticos entre los valores observados y los calculados no debe ser el único criterio que guíe la interpolación de datos climáticos. En este trabajo, la interpolación mediante Kriging da, al mismo tiempo, un buen ajuste estadístico (tabla 7) y una superficie suavizada (figura 9).

La correlación entre los datos calculados y los observados para las estaciones control (el 30\% del total) ofrece unos errores medios absolutos entre 2,7 mm para el mes julio y 9,8 para diciembre (tabla 7 ). El error medio es de signo positivo en todos los meses menos febrero, lo que significa que el modelo, como media, subestima la precipitación, aunque en cifras por debajo de los $1,5 \mathrm{~mm}$ mensuales. Las ordenadas en el origen por encima de cero indican que el modelo sobreestima las precipitaciones para los puntos con menos lluvia, aunque las pendientes por debajo de uno compensan esta sobrestimación con una subestimación de las precipitaciones más altas, como puede observarse en la figura 10. En esta figura se observa también como el modelo funciona mucho mejor para los valores de precipitación por debajo de $750 \mathrm{~mm}$ (menor dispersión de los datos), más frecuentes en las zonas llanas de la península, donde se encuentra el mayor número de estaciones meteorológicas.

\section{Temperatura}

La interpolación de las temperaturas resultó bastante diferente a la de las precipitaciones. El factor altitud explica la mayor parte de la variabilidad de 
las temperaturas a lo largo de la península ibérica ( $R^{2}$ entre 55 y 70$)$ excepto para los meses estivales, mayo y septiembre (figura 7). En estos meses es necesario añadir otras variables para aumentar el grado de ajuste, siendo las más importantes la radiación y las coordenadas geográficas (tabla 4). El efecto de la radiación tiene que ver con el carácter continental del clima de una buena parte del territorio peninsular, que se manifiesta más en los meses centrales del año (Capel, 2000; Font Tullot, 2000). La presencia de cielos despejados hace que la radiación haga calentarse intensamente el interior peninsular, alejado de la acción dulcificadora de los mares circundantes. En el mismo sentido, la distancia lineal a la costa muestra una correlación positiva con las temperaturas medias, particularmente en los meses estivales. La distancia orográfica, que mide el efecto combinado de la distancia a la costa y la altitud, muestra una correlación negativa con las temperaturas medias.

Las coordenadas geográficas influyen en el modelo de distinta forma. La coordenada latitud (Y) tiene todos los meses un signo negativo, en consonancia con la disminución de la temperatura hacia el norte (a mayor latitud). Sin embargo la influencia de la longitud (X) es menos importante y variable a lo largo del año. Resulta negativa para los meses más fríos (disminución de las temperaturas hacia el este), influida por una mayor altitud media en hacia el este, y positiva para los meses cálidos (aumento de las temperaturas hacia el este), debido al aumento del calor del Mediterráneo que afecta más a las tierras orientales de la península. La diferencia entre la altitud puntual y la altitud media, introducida en el modelo para tratar de reflejar las inversiones térmicas, solo resulto significativa en 6 de los 12 meses del año y con un peso menor que el de las otras variables (tabla 4). Esto puede deberse o bien a que no sea la variable más adecuada para medir las inversiones térmicas o a que la distribución de las estaciones no permita captar suficientemente bien este efecto.

La correlación espacial de los residuos de las regresiones de temperaturas queda patente por su ajuste a los variogramas modelo (tabla 6). Sin embargo, estos variogramas tienen un mayor efecto pepita que los de precipitación, lo que indica una mayor proporción de variabilidad espacial debida a variaciones aleatorias y relacionadas con la menor densidad de los datos.

En la validación de los modelos de temperatura, los modelos de regresión múltiple explicaban la mayor parte de la variabilidad, aumentando solo ligeramente el ajuste cuando se interpolaban los residuos de las regresiones (tabla 8), algo observado en otros trabajos para la España peninsular (Bustamante, 2003). Esto muestra la menor complejidad de los modelos de temperatura, algo observado por otros autores (Goodale et al., 1998; Agnew y Palutikof, 2000; Ninyerola et al., 2007a; Ninyerola et al., 2007b). 
La utilización de la radiación real mejora notablemente el modelo de temperaturas, sobre todo para los meses centrales del año. Utilizando este tipo de radiación no solo se consigue que esta variable resulte significativa en 8 de los 12 meses del año sino que entre los meses de abril y agosto su peso en el modelo sea realmente importante (tabla 4). Otros autores que han utilizado la radiación potencial no han observado que sea significativa en las temperaturas medias en modelos de regresión multivariante (Vicente-Serrano et al., 2003; Ninyerola et al., 2007b). La radiación real, medida desde los satélites, y la corregida con el factor topográfico, resultan significativas para los mismos meses y presentan pesos similares. Sin embargo el segundo tipo de radiación puede resultar mucho más apropiado para zonas heterogéneas, con muchas vertientes y diferentes pendientes y orientaciones, como la mayoría de los ambientes montañosos de la península ibérica. La radiación medida por los satélites Meteosat corresponde a una superficie plana y su efecto en el modelo multivariante de las temperaturas será igual, por ejemplo, para vertientes orientadas al norte o al sur, cuando las segundas son en realidad más cálidas. Por estas razones la radiación elegida finalmente en el modelo de temperaturas ha sido la corregida.

\section{Datos estimados y contexto climático actual}

Como cabría de esperar, el modelo climático presenta ciertos errores en la evaluación de las precipitaciones y temperaturas actuales (tablas 10 y 11) debido a los cambios más recientes del clima. En este sentido, la precipitación anual de las décadas más recientes ha disminuido apreciablemente en relación a las décadas de los 60 y 70 (Bladé et al., 2010), mientras que el aumento de las temperaturas, que se viene dando a lo largo de todo el siglo $\mathrm{XX}$, ha sido especialmente acusado entre 1973-2015, afectando este incremento más a las temperaturas de primavera y verano (Brunet et al., 2006).

La variación de los valores de precipitación y temperatura en los tres periodos climáticos referenciales está en consonancia estos cambios (tabla 9). Así, la disminución de las precipitaciones de los 60 Observatorios Principales entre el periodo 1961-1990 y 1981-2010 es considerable (casi $40 \mathrm{~mm}$ en las precipitaciones anuales). Esta disminución se ha dado sobre todo en invierno y primavera, y no ha sido compensada por el ligero ascenso de la precipitación otoñal (ver valores del mes de octubre). En el caso de las temperaturas el cambio es de 0,7 grados en la media anual, habiendo contribuido sobre todo el ascenso de las temperaturas de los meses centrales del año. 
Aunque las series de precipitación y temperatura utilizadas para elaborar el modelo abarcan el periodo 1961-2003, el número de estaciones que presenta datos para la década de los 60 es muy bajo, particularmente para la temperatura. Por tanto, los datos utilizados en el modelo se encuadran principalmente en el periodo 1970-2000 y deberían ser los datos de este periodo los que más se ajustaran al modelo climático calculado. En el caso de la precipitación, esto no ocurre así debido a que el modelo subestima las precipitaciones más altas pero sobreestima las bajas (tabla 7 y figura 10), como son las de los Observatorios Principales, situados en zonas llanas, normalmente alejadas de las montañas y, por tanto, con menor precipitación. Además de sobreestimar las precipitaciones del periodo 1971-2000, sobreestima en mayor medida las del periodo siguiente, más bajas por efecto del cambio climático, con la única excepción del mes de octubre (el único en el que han ascendido las precipitaciones en las últimas décadas).

Respecto a las temperaturas estimadas con el modelo, éstas coinciden bastante mejor con los valores registrados en el periodo 1971-2000, sobreestimando las del periodo 1961-1990 y subestimando las de 1981-2010 (tabla 11), lo que está en consonancia con el ascenso que se ha producido en las temperaturas a lo largo de los tres periodos climáticos.

\section{Conclusiones}

El modelo climático elaborado presenta las ventajas de haberse realizado con software libre a partir de un conjunto de datos de acceso público. Los resultados finales mejoran en algunos aspectos frente a modelos de interpolación anteriores. Por ejemplo, en el caso de la precipitación, la interpolación de los residuos mediante kriging produce superficies finales homogéneas, sin cráteres ni picos. Respecto a la temperatura, el modelo presenta la ventaja de incluir el efecto de una variable tan importante como la radiación para 8 meses del año, con un peso especialmente alto en los meses centrales del año. Dados los cambios climáticos acaecidos en los últimos años, el modelo presenta errores a la hora de predecir los valores actuales, y en líneas generales sobrestima las precipitaciones y subestima las temperaturas. Esta es una característica que hay que tener en cuenta a la hora de utilizar los mapas obtenidos.

NOTA: los mapas obtenidos pueden descargarse en el blog «Bosques ibéricos: geografía, ecología y transformación antrópica», cuya dirección es: http://ecohistoriabqs.blogspot.com.es/2016/11/mapas-climaticos.html 


\section{BiBLIOGRAFÍA}

Agnew, M.D. y Palutikof, J.P. (2000) GIS-based construction of baseline climatologies for the Mediterranean using terrain variables. Climate research, 14: 115-127.

Barry, R.G. (1992) Mountain weather and climate. Routledge, London, 402 pp.

Bladé, I., Cacho, I., Castro-Díez, Y., Gomis, D., González-Sampériz, P., Miguez-Macho, G., Perez, F.F., Rodríguez-Fonseca, B., Rodríguez-Puebla, C., Sánchez, E., Sotillo, G., Valero-Garcés, B. y Vargas-Yáñez, M. (2010) Tendencias atmosféricas en la península ibérica durante el periodo instrumental en el contexto de la variabilidad natural. En: F.F. Pérez y R. Boscolo (Editores), Clima en España: pasado, presente y futuro. Informe de Evaluación del Cambio Climático Regional. CLIVAR (Climate variability and predictability) España, pp. 25-42.

Brown, D.P. y Comrie, A.C. (2002) Spatial modeling of winter temperature and precipitation in Arizona and New Mexico, USA. Climate research, 22: 115-128.

Brunet, M., Saladié, O., Jones, P.D., P.D., J.S., Aguilar, E., Moberg, A., Lister, D., Walther, A., López, D. y Almarza, C. (2006) The development of a new dataset of Spanish daily adjusted temperature series (SDATS) (1850-2003). International Journal of Climatology, 26(13): 1777-1802.

Bustamante, J. (2003) Cartografía predictiva de variables climáticas: comparación de distintos modelos de interpolación de la temperatura en España peninsular. Graellsia, 59(2-3): 359-376.

Capel, J.J. (2000) El clima de la península ibérica. Ariel, Barcelona, 281 pp.

Chapman, L. y Thornes, J.E. (2003) The use of geographical information systems in climatology and meteorology. Progress in Physical Geography, 27(3): 313-330.

Cressie, N.A.C. (1993) Statistics for Spatial Data. John Wiley \& Sons, New York, 416 pp.

Daly, C. (2006) Guidelines for assessing the suitability of spatial climate data sets. International Journal of Climatology, 26: 707-721.

Daly, C., Gibson, W., Taylor, G., Johnson, G. y Pasteris, P. (1994) A statisticaltopograpbic model for mapping climatological precipitation over mountainous terrain. Journal of Applied Meteorology, 22: 99-113.

Font Tullot, I. (2000) Climatología de España y Portugal. Universidad de Salamanca, Salamanca, $422 \mathrm{pp}$.

Funk, C., Michaelsen, J., Verdin, J., Artan, G., Husak, G., Senay, G., Gadain, H. y Magadazire, T. (2002) The collaborative historical African rainfall model: description and evaluation. International Journal of Climatology, 1: 47-66.

Goodale, C.L., Aber, J.D. y Ollinger, S.V. (1998) Mapping monthly precipitation, temperature and solar radiation from Ireland with polynomial regression and a digital elevation model. Climate research, 10: 35-49.

Guan, H., Wilson, J.L. y Makhnin, O. (2005) Geostatistical mapping of mountain precipitation incorporating autosearched effects of terrain and climatic characteristics. Journal of Hydrometeorology, 6: 1018-1031. 
Hengl, T. (2009) A practical guide to geostatistical mapping. Open Access Publication: http://spatial-analyst.net/book/, Amsterdan, 270 pp.

Hengl, T., Heuvelink, G.B.M. y Rossiter, D.G. (2007) About regression-kriging: from theory to interpretation of results. Computers \& Geosciences, 33(10): 1301-1315.

Hevesi, J.A., Istok, J.D. y Flint, A.L. (1992) Precipitation estimation in mountainous terrain using multivariate geostatistics, Part 1: Structural Analysis. Journal of Applied Meteorology, 31: 661-676.

INM (2004) Guía resumida del clima de España (1971-2000). Direción General del Instituto Nacional del Meteorología, Madrid, 257 pp.

Ishida, T. y Kawashima, S. (1993) Use of cokriging to estimate surface air temperature from elevation. Theoretical and Applied Climatology, 47: 147-157.

ITC (2001) ILWIS 3.0 Academic. User's Guide. International Institute for Aerospace Survey and Earth Sciences (ITC), http://www.itc.nl/ilwis, Hengelosestraat (Paises Bajos), 530 pp.

Kurtzman, D. y Kadmon, R. (1999) Mapping of temperature variables in Israel: a comparison of different interpolation methods. Climate research, 13(1): 33-43.

Kyriakidis, P.C., Kim, J. y Miller, N.L. (2001) Geostatistical mapping of precipitation from rain gauge data using atmospheric and terraín characteristics. Journal of Applied Meteorology, 40: 1855-1877.

Martín Vide, J. y Olcina, J. (2001) Climas y tiempos de España. Alianza, Madrid, 258 pp.

Martínez-Cob, A. (1996) Multivariate geostatistical analysis of evapotranspiration and precipitation in mountainous terrain. Journal of Hydrology, 174: 19-35.

Miller, W. (2013) Statistics and measurement concepts with OpenStat. Springer, Nueva York, $237 \mathrm{pp}$.

Nalder, I. A. y Wein, R. W. (1998) Spatial interpolation of climatic Normals: test of a new method in the Canadian boreal forest. Agricultural and Forest Meteorology, 92: 211-225.

Neteler, M. y Mitasova, H. (2004) Open Source GIS: A GRASS GIS approach. Kluwer Academic Publishers, Nueva York, 401 pp.

Ninyerola, M., Pons, X. y Roure, J.M. (2000a) A methodological approach of climatological modelling of air temperature and precipitation through gis techniques. International Journal of Climatology, 20: 1823-1841.

Ninyerola, M., Pons, X. y Roure, J.M. (2000b) Monthly precipitation mapping of the Iberian Peninsula using spatial interpolation tools implemented in a Geographic Information System. Theoretical and Applied Climatology, 89: 195-209.

Ninyerola, M., Pons, X. y Roure, J.M. (2007a) Monthly precipitation mapping of the Iberian Peninsula using spatial interpolation tools implemented in a Geographic Information System. Theoretical and Applied Climatology, 89(3-4): 195-209.

Ninyerola, M., Pons, X. y Roure, J. M. (2007b) Objective air temperature mapping for the Iberian Peninsula using spatial interpolation and GIS. Int J Climatol, 27(9): 1231-1242.

Perry, M. y Hollis, D. (2005) The generation of monthly gridded datasets for a range of climatic variables over the UK. International Journal of Climatology, 25: 1041-1054. 
Phillips, D.L., Dolph, J. y Marks, D. (1992) A comparison of geostatistical procedures for spatial analysis of precipitation in mountainous terrain. Agricultural and Forest Meteorology, 58(119-141).

Sharples, J.J., Hutchinson, M.F. y Jellet, D.R. (2005) On the horizontal scale of elevation dependence of Australian monthly precipitation. Journal of Applied Meteorology, 44.: 1850-1865.

Smith, R.B. (1979) The influence of mountains on the atmosphere. Advances in Geophysics, 21: 87-230.

Tveito, O.E. (2008) Spatialisation of climatological and meteorological information with the support of GIS. En: O.E. Tveito, M. Wegehenkel, F.v.d. Wel y H. Dobesch (Editores), The Use of Geographic Information Systems in Climatology and Meteorology. COST Office-European Communities, Brussels, pp. 36-163.

Vicente-Serrano, S.M., Saz-Sánchez, M.A. y Cuadrat, J.M. (2003) Comparative analysis of interpolation methods in the middle Ebro Valley (Spain): application to annual precipitation and temperature. Climate research, 24: 161-180.

Willmott, C. (1982) Some comments on the evaluation of model performance. Bulletin of the American Meteorological Society, 63(11): 1309-1313.

Willmott, C., Robeson, S.M. y Janis, M.J. (1996) Comparison of approaches for estimating time-averaged precipitation using data from the USA. Int J Climatol, 16(10): 1103-1115.

Xin, Y., Guoan, T., Chenchao, X. y Fengdong, D. (2007) Terrain revised model for air temperature in mountainous area based on DEMs: A case study in Yaoxian county. Journal of Geographical Sciences, 17(4): 399-408.

Fecha de recepción: 1 de diciembre de 2016.

Fecha de aceptación: 6 de junio de 2017. 